\title{
Enhancing Experience Prototyping by the help of Mixed-Fidelity Prototypes
}

\author{
Ansar-UI-Haque Yasar \\ Linköping University \\ Sweden \\ Ph: (+46) 704035216 \\ ansya448@student.liu.se
}

\begin{abstract}
In this research review I undertook the problem related to the usage of a new concept known as the Mixed- Fidelity Prototype which is a mixture of its predecessors Low- and High- Fidelity Prototypes in Experience Prototyping. Experience Prototyping is a good way to explore, communicate and interact with the designs we develop like experiencing cycling on the ice, although the mood, snow conditions, bicycle type and many other factors really matter and tend to change with time. Experience Prototyping in itself is a very large domain to be explored separately but this research contains the idea to improve the ways we can achieve these factors with a minimal variation. Prototyping has the involvement of both the users and the stake holders so it is really important that correct experiments and the prototypes should get the desired results. With the effective usage of mixed-fidelity prototype in Experience Prototyping a better understanding of the real system can be developed as it gives a feeling of both the Low- and High- Fidelity Prototypes. It can unable an HCI developer to properly identify the requirements either functional or non-functional as identified by the proposed user. This paper has a comparison study between the uses of different prototyping models in Experience prototyping for better understanding of the system under development in detail and reasons for preferring Mixed-Fidelity Prototypes over the others.
\end{abstract}

\section{Keywords}

Prototyping, low-fidelity, high-fidelity, mixedfidelity, experience prototyping, methods.

Permission to make digital or hard copies of all or part of this work for personal or classroom use is granted without fee provided that copies are not made or distributed for profit or commercial advantage and that copies bear this notice and the full citation on the first page. To copy otherwise, or republish, to post on servers or to redistribute to lists, requires prior specific permission and/or a fee. MC'07 (Mobility'07), September 10-12, 2007, Singapore.

Copyright 2007 ACM 978-1-59593-819-0......\$5.00

\section{INTRODUCTION}

The term Prototype means an early model of a product in development for better understanding. The Prototypes helps in better understanding the product which is yet to be developed, it also helps in understanding the functionalities the actual product may perform, the way real product should look, feel and many other related things. "Prototyping methods are widely recognized as an important component of the HCI process. When correctly applied, the ability of a prototype to identify and correct potential problems early in the development cycle can pay for the cost of the prototype many times over" [3]. It can also be referred as "Prototypes are widely recognized to be a core means of exploring and expressing designs for interactive computer artifacts. It is common practice to build prototypes in order to represent different states of an evolving design, and to explore options. However, since interactive systems are complex, it may be difficult or impossible to create prototypes of a whole design in the formative stages of a project. Choosing the right kind of more focused prototype to build is an art in itself, and communicating its limited purposes to its various audiences is a critical aspect of its use.'[4]

This paper is based on the ideas presented by different researchers $[1,2,3,4]$ in the field of Low-, High- and Mixed-Fidelity Prototypes to be used in Experience prototyping. In general the LowFidelity prototypes refer to as the paper based prototypes in which the initial workflow model of the systems which is yet to be developed, a HighFidelity prototype then refers to a better Graphics model of a product which is yet to be developed using some tools like VB, Flash, C\#, etc, a MixedFidelity prototype as proposed by the author [3] is referred to as a model "which is high-fidelity in some respects and low-fidelity in others". The Experience prototyping which is according to the 
author [2] described as "the look and feel of the system i.e. whatever the user feels and hears while using it". The thing which makes Experience prototyping important is that way these days we being developers are so much involved in trying to make more and more complex systems making it more difficult for ourselves in the understanding the real requirements so in order to understand those requirements a real user interaction is needed who can identify the real demands based on the "integrated and holistic experiences set in context" or "a form of prototyping that enables design team members, users and clients to gain first-hand appreciation of existing or future conditions through active engagement with prototypes." [2].

This is paper is divided into six different sections giving details of the paper. Section 2 contains the description about the topic and explanation of the methods used for comparison, Section 3 contains some Experiments or Tests being performed, Section 4 is the Conclusion and the future work, Section 5 is the acknowledgements and the last but not the least Section 6 is about references.

\section{DESCRIPTION}

It's really important here to expedite about the designers, users and engineer's use of prototypes as model for what is to be built. A designer will treat a prototype either Low-, High- or MixedFidelity in order to identify the functionalities and requirements of a system under development. An engineer's use the prototypes so that they can test the effectiveness of any functionality being developed for a system and identified by a designer. A user perspective comes in the last which is to get a better understanding of a system i.e. its usefulness and functionalities using the prototype(s) developed by the engineers.

Before starting off with Experience Prototyping, the author's [2,7] analysis and research has shown that experience prototyping has a important part in the three ways:

"By helping to develop understand about the essence or essential factors of an existing experience: Experience Prototyping simulates important aspects of the whole or parts of the relationships between people, places and objects as they unfold over time."

"In exploration and evaluation of ideas: Experience Prototyping can provide inspiration, confirmation or rejection of ideas based upon the quality of experience they engenders. It produces answers and feedback to designers' questions about proposed solutions in terms of what would it feel like if..."

"In communication of issues and ideas: by enabling other to engage directly in a proposed new experience it provides common ground for establishing a shared point of view"

This tells us that the Experience prototyping is not just a tool or some gadget, it is in real about focusing on the design problems for an effective solution. In experience prototyping hybrid skills are needed which is in a sense very good as one does not have to be specific and this way it's a mixture of multiple or more design principles.

Now a problem arises that which method or fidelity prototype should be used in Experience Prototyping for achieving better results. Another important aspect is that "What do prototypes prototype?" The author's [4] describe this concept using a figure 1. This figure tells us that a prototypes prototype three major things: (1) "What role will the device / product being developed play in a users life?" (2) "How the device / product should look and feel?" and (3) "How should the device / product be implemented?" [4]. Using these three key facts developers can get the prototypes to convey their purpose and for a better implementation of the prototype into reality.

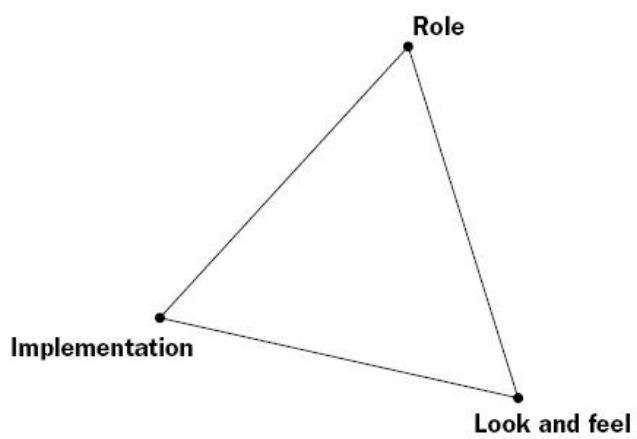

FIGURE - 1: What do Prototypes Prototype? [4]

The fidelity types are Low-, High- or MixedFidelity Prototypes. Each fidelity type is discussed in detail as under:

\subsection{Low-Fidelity Prototypes in Experience Prototyping}

Let us consider a Low-Fidelity Prototype in experience prototyping. A low-fidelity prototype 
is also known as a paper-prototype as it is implemented using pen and paper or "described within a spectrum of fidelity with lowfidelity, ostensibly low-cost methods such as whiteboard or paper sketches at one end" [3]. "Low-fidelity methods have received a great deal of recognition in the field for their ability to validate designs and predict large problems at an extremely low cost" [3]. It is very low level and easy to implement for the developers but on the other hand if it is related to the users / client end a low-fidelity model is not so good in communicating the timing issues. "It is good and effective throughout the product development cycle, not just during the initial stages of design" $[1,5]$. This is due to that the real look and feel feature cannot be achieved in a true sense and the user / client cannot play with it as in reality.

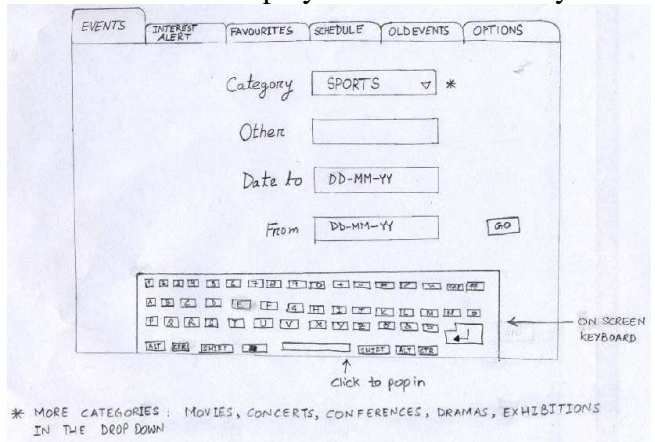

FIGURE -2: A Typical Low-Fidelity Prototype of an Event Manager.

In the Figure -2, a user will have a good overview about an interface of an Event Manager and even he can play with it using a story board with more paper descriptions of the product but can never feel the real response, the time of the response, some unexpected behaviour, etc. When discussing about Experience Prototyping in the context of Low- Fidelity prototypes we can say that in the case of the Event Manager as shown in the figure above a designer, user or an engineer can not fully interact and communicate ideas as it is paper based. The idea about the interface i.e. "look" of the Event Manager can be judged but the "feel" of the system can not be identified easily. Hence, experience prototyping can not be felt in the real sense if using low-fidelity prototype for it.

\subsection{High-Fidelity Prototypes in Experience Prototyping}

A High-Fidelity Prototype is considered to be an advanced prototype as it is developed using some tools and languages or "High-fidelity methods, on the other hand, have received passing recognition for their ability to convince management or other stakeholders that due diligence has been given to the product design and that the real thing is indeed on its way. However, beyond this grudging recognition, high-resolution methods are often dismissed as being too cumbersome, too expensive to develop, or too likely to set unrealistic expectations in the minds of users and customers" $[3,5,7]$. The High-Fidelity Prototypes are somehow not so cost efficient as the usage of tools and different high tech equipment involves a lot of cost in it. In any case a High-Fidelity prototype helps us in "understanding, exploring and evaluating design phases" [2] as a user / client can have a better idea about the product as the client / user can play with it. The user / client will get some how more idea about the input responses and the efficiency of the product.

High-fidelity models, like shown in figure 3, where having some good features can also have some negative impacts as well. The high-fidelity models are very expensive and not all cost effective to be built so for a small system the cost factor will increase to a great extent. When discussing about Experience Prototyping in the context of High- Fidelity prototypes we can say that in the case of the Digital Camera as shown in the figure below a designer, user or an engineer can fully interact and communicate ideas as it is somehow reality based and very close to the real thing. The idea about the interface i.e. "look" of the Digital Camera can be judged along with the "feel" of the system. So experience prototyping will be very good in case of high-fidelity prototypes but a very important factor i.e. cost will increase to a greater limit.

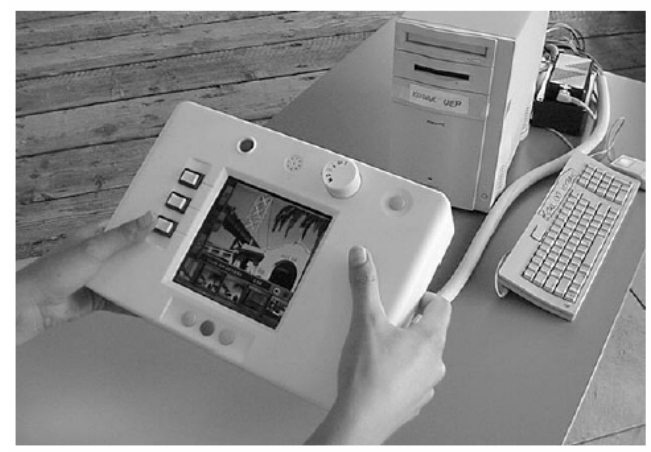

FIGURE -3: A Digital Camera Prototype. [2] 


\subsection{Mixed-Fidelity Prototypes in Experience Prototyping}

Now comes the turn of the Mixed-Fidelity Prototypes which is a low-fidelity in some aspects and high-fidelity on some other aspects. This type of prototyping is suggested by authors' [3] which is very cost effective as only the parts in highfidelity may affect the cost whereas the parts which do not need expensive prototyping are paper based and cheap. This is in a sense very reasonable to use with Experience Prototyping as it may save the cost along with that the designer, user / client or engineer can also have a better understanding of the product.

The Mixed-Fidelity Prototypes are getting famous and more in use these days by the developers of HCI. Hence, Mixed-Fidelity Prototypes can be the best way to communicate ideas and interact with them as well which is the core purpose of Experience Prototyping.

\section{TESTS AND PROOFS}

Authors [1,2,3,4] performed many tests and experiments in order to identify the pros and cons for the fidelity prototypes. Some experiments have shown many interesting results.

One of the experiment which authors' [3,6] performed was related to the Mixed-Fidelity Prototypes which lead to Break the fidelity bearer. The authors' $[3,6]$ "SPIFe prototype for mission planning tools is an example of a mixed-fidelity prototype that incorporates a high-fidelity data model and level of interactivity, but a low level of visual refinement and fairly narrow functionality". It is an excellent example to develop a prototype by saving resources and providing visual interface along with the functionality depth which can be applied easily by the developers and is also useful for the users / clients. The experiment as described by the author is "It was decided at an early stage to use actual mission data - the richest data available - to populate this prototype in order to provide a realistic environment and scale. Also, since upcoming missions are largely staffed with people with experience on MER, populating the prototype with MER data tended to make it not only realistic but also familiar to user-test subjects and mission management. Admittedly, this decision was easy in this case; it was not a prohibitively costly approach. Due to their involvement with MER operations, the designers and prototype developer were fortunate in having access to this data set and an understanding of it. They were able to reuse planreading software from the Constraint Editor, an operational tool that is in use on MER. The SPIFe prototype is web-based, and thus takes advantage of some of the interactive display capabilities available in Firefox and other browsers compliant with the DOM and CSS standards. This also simplifies installation for demos, since Firefox runs on three ubiquitous computer platforms and is widely installed. The client-side scripts are implemented in JavaScript. All of the HTML, and the variable data for JavaScript, are generated dynamically from actual MER data by a server-side Lisp program. This approach allows designers to choose from well over a thousand actual plans for a one-time programming cost. In contrast, with a handcrafted paper or PowerPoint prototype, the cost would be proportional to the depth of the data model. Maintaining state, such as the effect of moving activities around the time timeline, was tricky in a web application, but that problem was solved early on, and the prototype can be used for end-to-end tasks; that is, it provides unlimited depth of functionality at no further development cost, once again in contrast to a hand-crafted prototype. Due to reuse of existing code and the decision to focus on particular axes of fidelity, the SPIFe prototype was created by a single developer in approximately one month.

Although it would be possible to simulate some aspects of the interface using traditional lowfidelity means, for example using acetate overlays to indicate highlighting, the cost of building such a prototype to handle diverse scenarios would have been large. Additionally, due to physical limitations on how quickly such an prototype can be manipulated, it would not have been possible to collect the timing data desired.

While this prototyping effort was underway, tool developers at NASA Ames and JPL realized that a unified, component-based approach to software development could yield an integrated suite of ground based mission tools for deployment on upcoming Mars surface missions. Many of the design solutions tested as part of the SPIFe prototype have been incorporated in to the new application, called Ensemble. Ensemble is being developed using the Java Eclipse development framework, which has allowed the team to rapidly realize concepts into working software components. The Ensemble application is 
necessarily "high fielity" in all five dimensions. It is the final product of the development process, and as such it sets a practical upper bound on each of the five dimensions. For example, the Eclipse framework provides a highly refined, platform appropriate look-andfeel, giving Ensemble a high level of visual refinement. Additionally, Ensemble developers have already incorporated many of the interactive behaviors from the SPIFe prototype, lending it a high level of interactivity.

During the Mars Exploration Rover (MER) mission, operations personnel used a planning tool called the Mixedinitiative2 Activity Plan GENerator, or MAPGEN, to schedule rover activity. The fundamental task of planning consists of selecting activities requested by mission scientists (e.g. "capture an image of rock $x$ " or "drive to location $y$ ") and assigning them execution times. This assignment requires users to pay close attention to rover resources and can get orders of magnitude more complex for every activity added to the plan.” [3]

The results of this experiment showed "The mixed-fidelity prototype has a few properties that made this experiment possible. First, since the level of interactivity was set high, the designers can be assured that the timing data is accurate to within a few milliseconds - plenty of precision given the multiple-second differences between the tools. Second, since the prototype uses a very high-fidelity data model - in fact it reads the exact same plan files that the other two tools do - it was possible to ask the same set of questions (randomized between subject) in all three conditions. Since the other dimensions of the prototype were left low-fidelity, it remains lightweight and easy to change based on ongoing experiment or user test data." [3]. The test results showed that Mixed-Fidelity (MAPGEN) prototype showed no difference in results with a High-Fidelity (Ensemble/SPIFe) prototype whereas it was better in saving cost. The mixedfidelity prototypes are yet proved to be good for market oriented and team related studies achieving the desired data and functionality from the outer world but still very much applicable and result efficient.

\section{CONCLUSION AND FUTURE WORK}

My current research and survey shows that a Mixed-Fidelity Prototype should be used in Experience Prototyping by the designer world and engineers which can help them in better representation of their ideas, views and understandings.

I think that even after this there is still a lot of room available for more research in this area of Mixed-Fidelity and Experience Prototyping as the designers are still not $100 \%$ sure that which part should be high level and which should be low level in a mixed fidelity prototype. Along with this problem in Experience prototyping we cannot always get the real need and the idea or communicate or identify the ideas of the other people or the real user which creates a lot of space for other types of prototyping methods yet to be developed.

Finally I think for getting Effective results the usage of Mixed-Fidelity Prototype in Experience Prototyping may help during the building process of a system and also exemplifies how traditional and more passive communication techniques and Experience Prototyping can work hand-in-hand, with the goal of sharing a new user experience with an audience.

\section{ACKNOWLEGDEMENTS}

Author would like to acknowledge the help provided by my teachers Mr. Nils Dahlbäck and Mr. Per Söker for making this research successful. Without their kind cooperation and their lectures about writing scientific papers and System Usability Issues, it would have been difficult for me to complete this paper.

\section{REFERENCES}

[1] Robert A. Virzi, Jeffrey L., Sokolov and Demetrios Karis. Usability Problem Identification Using Both Low- and High-Fidelity Prototypes, in the Proceedings of CHI 96, pp. 236-243, April 13-18, 1996.

[2] Marion Buchenau and Jane Fulton Suri. Experience Prototype, in the Proceedings of ACM DIS '00, pp. 424-433, 2000.

[3] Michael McCurdy, Christopher Connors, Guy Pyrzak, Bob Kanefsky and Alonso Vera. Breaking the Fidelity Barrier: An Examination of our Current Characterization of Prototypes and an Example of a Mixed-Fidelity Success, in the Proceedings of ACM CHI 2006, pp. 1233-1242, April 22-27, 2006.

$472 \quad$ Proc. of the $4^{\text {th }}$ Intl. Conf. on Mobile Technology, Applications and Systems (Mobility 2007) 
[4] Stephanie Houde and Charles Hill. What do Prototypes Prototype?, based on the article by Houde, S., and Hill, C., What do Prototypes Prototype?, in the Handbook of HCI ( ${ }^{\text {nd }}$ Ed.), Elsevier Science B.V: Amsterdam, 1997.

[5] Rudd, J., Stern, K., Isensee, S. Low vs High-fidelity prototyping debate, interactions, v.3 n.1, p.76-85, Jan 1996.
[6] Garret, J.J., Ajax: A New Approach to Web Applications. http://www.adaptivepath.com/publicatio ns/essays/archives/000385.php, 2005

[7] Landay, J.A., Myers, B.A. Sketching Interfaces: Toward More Human Interface Design, Computer, v.34 n.3, p.56-64, March 2001. 